\title{
Dynamic CT for Parathyroid Disease: Are Multiple Phases Necessary?
}

P. Raghavan, C.R. Durst, D.A. Ornan, S. Mukherjee, M. Wintermark, J.T. Patrie, W. Xin, A.L. Shada, J.B. Hanks, and P.W. Smith

\begin{abstract}
BACKGROUND AND PURPOSE: A 4D CT protocol for detection of parathyroid lesions involves obtaining unenhanced, arterial, early, and delayed venous phase images. The aim of the study was to determine the ideal combination of phases that would minimize radiation dose without sacrificing diagnostic accuracy.
\end{abstract}

MATERIALS AND METHODS: With institutional review board approval, the records of 29 patients with primary hyperparathyroidism who had undergone surgical exploration were reviewed. Four neuroradiologists who were blinded to the surgical outcome reviewed the imaging studies in 5 combinations (unenhanced and arterial phase; unenhanced, arterial, and early venous; all 4 phases; arterial alone; arterial and early venous phases) with an interval of at least 7 days between each review. The accuracy of interpretation in lateralizing an abnormality to the side of the neck (right, left, ectopic) and localizing it to a quadrant in the neck (right or left upper, right or left lower) was evaluated.

RESULTS: The lateralization and localization accuracy ( $90.5 \%$ and $91.5 \%$, respectively) of the arterial phase alone was comparable with the other combinations of phases. There was no statistically significant difference among the different combinations of phases in their ability to lateralize or localize adenomas to a quadrant ( $P=.976$ and .996 , respectively).

CONCLUSIONS: Assessment of a small group of patients shows that adequate diagnostic accuracy for parathyroid adenoma localization may be achievable by obtaining arterial phase images alone. If this outcome can be validated prospectively in a larger group of patients, then the radiation dose can potentially be reduced to one-fourth of what would otherwise be administered.

ABBREVIATIONS: NPV = predictive value; PPV = positive predictive value

$\mathbf{P}$ rimary hyperparathyroidism is defined as hypercalcemia secondary to overproduction of parathyroid hormone by $\geq 1$ parathyroid gland. ${ }^{1}$ Excessive unregulated parathyroid hormone production by a parathyroid adenoma or hyperplasia results in increased renal absorption of calcium, increased synthesis of $1,25(\mathrm{OH})_{2} \mathrm{D}_{3}$, phosphaturia, and accelerated bone resorption. ${ }^{2}$ Primary hyperparathyroidism can only be cured by surgical exci-

Received November 12, 2013; accepted after revision March 17, 2014.

From the Department of Diagnostic Radiology and Nuclear Medicine (P.R.), University of Maryland Medical Center, Baltimore, Maryland; and Departments of Radiology (C.R.D., D.A.O., S.M., M.W., J.T.P., W.X.) and Surgery (A.L.S., J.B.H., P.W.S.), University of Virginia, Charlottesville, Virginia.

The study was conducted at the University of Virginia Health System, 1215 Lee St, Charlottesville, Virginia 22908.

Please address correspondence to Prashant Raghavan, MBBS, Division of Neuroradiology, Department of Diagnostic Radiology and Nuclear Medicine, University of Maryland School of Medicine, 22 S Greene St, Baltimore, MD 21201; e-mail: prashant.raghavan@gmail.com

三 Indicates article with supplemental on-line tables.

Indicates article with supplemental on-line figures.

http://dx.doi.org/10.3174/ajnr.A3978 sion of the abnormal parathyroid tissue, which, in approximately $90 \%$ of patients, is a solitary adenoma. With the advent of improved preoperative imaging and the use of intraoperative parathyroid hormone monitoring, standard cervical (or "4-gland") exploration has given way to "minimally invasive" or directed parathyroidectomy for the treatment of single-gland disease in the hands of most experienced parathyroid surgeons. ${ }^{3}$ Directed parathyroidectomy allows a smaller surgical incision and a more limited cervical dissection, thereby decreasing the risk of recurrent laryngeal nerve injury, permanent hypoparathyroidism, and postoperative pain; decreasing the cost and duration of hospital stay; and, in some patients, avoiding general anesthesia. ${ }^{3}$

Preoperative localization may be achieved with sonography, technetium Tc99m sestamibi imaging (with or without SPECT/ CT), and/or dynamic 4D CT. On a standard, nondynamic contrast-enhanced CT of the neck with scan delays approximately 80 seconds after injection, however, differentiation between parathyroid lesions and adjacent small lymph nodes may not be possible. Reported sensitivities of standard neck CT range from $46 \%$ to $87 \%$ for localization of parathyroid adenoma. ${ }^{4} 4 \mathrm{D} \mathrm{CT}$, intro- 
duced by Rodgers et al, ${ }^{3}$ involves obtaining precontrast, arterial, venous, and delayed images of the neck after administration of a bolus of intravenous contrast. 4D CT has been reported to be superior to sonography and sestamibi scanning, with sensitivities in excess of $90 \%$ for lateralizing a parathyroid adenoma to one side of the neck and in excess of $85 \%$ for localizing an abnormal gland to the correct quadrant of the neck. ${ }^{5,6}$ In comparison, the sensitivity for precise localization to a quadrant of the neck ranges from $33 \%$ to $65 \%$ by using sestamibi and $29 \%-57 \%$ for sonography. ${ }^{3}$

The principle behind 4D CT is that the hypervascularity of parathyroid lesions results in rapid enhancement (detectable in the arterial phase) and washout of contrast (detectable on venous and delayed phases) compared with lymph nodes, which enhance to a lesser degree and retain contrast through the venous and delayed phases. Despite the appearance of numerous articles on the subject in the literature, there is not yet a consensus on what constitutes an optimal dynamic CT study: Some authors advocate 2 phases (unenhanced, arterial), some advocate 3 phases (unenhanced, arterial, venous), some advocate 2.5 phases (unenhanced and arterial phase of the neck followed by a venous phase of the lower neck and entire chest, with a single contrast bolus), while others support 4 phases (unenhanced, arterial, venous, and delayed). ${ }^{3,5,7-10}$ Given the variability in techniques reported in the literature, our objective was to determine the optimal dynamic CT protocol necessary for accurate detection of these lesions in a selected cohort of patients undergoing surgical exploration for primary hyperparathyroidism.

\section{MATERIALS AND METHODS}

This study was approved by our institutional review board. Data were collected retrospectively and de-identified in compliance with Health Insurance Portability and Accountability Act regulations.

\section{Patient Selection}

Between May 2008 and March 2010, 103 patients underwent surgical exploration for primary hyperparathyroidism. Of these, 29 patients met the following inclusion criteria:

1) Laboratory data (blood parathyroid hormone, calcium, inorganic phosphorus, and $1,25[\mathrm{OH}]_{2} \mathrm{D}_{3}$ levels) consistent with primary hyperparathyroidism.

2) Preoperative 4D CT was performed either because of equivocal sonography and sestamibi imaging findings or to facilitate surgical planning.

The clinical records of all patients were reviewed to identify sonography and sestamibi results. Eighteen patients underwent sonography, and 16 underwent sestamibi scanning. Of the 18 patients who underwent sonographic examinations in the clinic, 11 had findings that were negative or equivocal. Three accurately predicted the site of the lesion, and 4 incorrectly predicted the site of the lesion. The sensitivity for all 4 quadrants was 37\%, and the specificity was $92 \%$. Of the 16 patients in whom sestamibi imaging was performed, 7 studies had negative or equivocal findings. Seven lesions were accurately identified, and 2 were incorrectly identified. The sensitivity of sestamibi for all 4 quadrants was $37 \%$, and the specificity was $95 \%$. Six patients were re-operative
Table 1: Sequences that readers were allowed to use for interpretation each week

\begin{tabular}{cl}
\hline Week No. & \multicolumn{1}{c}{ Sequences } \\
\hline 1 & Unenhanced, arterial \\
2 & Unenhanced, arterial, venous \\
3 & Unenhanced, arterial, venous, delayed \\
4 & Arterial \\
5 & Arterial, venous \\
\hline
\end{tabular}

candidates. Each of these 6 patients underwent a preoperative sestamibi scan, and 4 of the 6 underwent preoperative sonography.

\section{CT Study}

Imaging was performed with a 32-section multidetector CT scanner. The scanning parameters were $120 \mathrm{kVp}, 50-400 \mathrm{~mA}$ range, 1 -second rotation speed, pitch of 0.968 , detector configuration section width of $1.25 \mathrm{~mm}$, total beam width of $40 \mathrm{~mm}$, and table translation speed of $38.75 \mathrm{~mm}$ per gantry rotation; $1.25-\mathrm{mm}$ thick sections were reconstructed at a window width of $350 \mathrm{HU}$ and a 40-HU window center with the detail reconstruction kernel. An unenhanced phase was first obtained. Twenty-five seconds before commencement of the arterial phase, $120 \mathrm{~mL}$ of iodinated contrast material (iohexol, Omnipaque 350; GE Healthcare, Piscataway, New Jersey) was injected at $4 \mathrm{~mL} / \mathrm{s}$ via a pressure injector. A third venous phase was obtained 50 seconds after the commencement of injection, and a fourth delayed phase was obtained 80 seconds after the beginning of injection. All scans were obtained from the carina to the angle of the mandible.

The volume CT dose index for the unenhanced phase was $26.45 \mathrm{mGy}$. For the other phases, it was $25.41 \mathrm{mGy}$. The doselength product (milligray $\times$ centimeter) for the unenhanced phase was 590.49, and for the other phases, it was 567.28.

\section{Image Analysis}

Four board-certified neuroradiologists (P.R., S.M., D.A.O., C.D.) with between 1 and 6 years' experience in neuroimaging, retrospectively reviewed each study. Each reader was blinded to the original radiology interpretation, surgical records, and pathology findings. During a 5-week period, the readers were asked to assess each study by using 5 different combinations of the available 4D CT sequences. The order and number of sequences available for interpretation each week were designed to determine whether additional sequences would improve the reader's accuracy in identification of a parathyroid lesion. All readers reviewed the same subset of sequences according to the schedule in Table 1. However, the order of patients was scrambled for each reader so that the readers would not be able to discuss their findings. Two methods were used to limit memory bias. First, the readers were asked to allow 1 week between each assessment. Second, the patients were de-identified, and the reading order was randomly generated and re-scrambled each week.

A lesion that demonstrated enhancement in the arterial phase and was present in one of the locations described below was considered compatible with a parathyroid lesion. The readers were asked to classify the lesions according to their anatomic location: left upper quadrant, left lower quadrant, right upper quadrant, or right lower quadrant with respect to a transverse plane through the middle of the thyroid gland and the midline, or ectopic when 
Table 2: Results of localization analysis by sequence combination

\begin{tabular}{|c|c|c|c|c|c|c|}
\hline Week & Sequences & $\begin{array}{c}\text { Accuracy \% } \\
(95 \% \mathrm{CI})\end{array}$ & $\begin{array}{c}\text { Sensitivity \% } \\
(95 \% \mathrm{CI})\end{array}$ & $\begin{array}{c}\text { Specificity \% } \\
\text { (95\% CI) }\end{array}$ & $\begin{array}{c}\text { PPV \% } \\
(95 \% \mathrm{CI})\end{array}$ & $\begin{array}{l}\text { NPV \% } \\
(95 \% \mathrm{CI})\end{array}$ \\
\hline 1 & $U, A$ & $91.2(86.5-94.4)$ & $82.1(74.8-88.1)$ & $94.1(91.5-96.1)$ & $81.6(74.2-87.6)$ & $94.3(91.7-96.3)$ \\
\hline 2 & $\mathrm{U}, \mathrm{A}, \mathrm{V}$ & $90.9(86.6-93.9)$ & $82.9(75.6-88.7)$ & 93.4 (90.7-95.5) & $80.0(72.6-86.2)$ & 94.5 (91.9-96.4) \\
\hline 3 & $U, A, V, D$ & $90.5(86.1-93.6)$ & $82.1(74.8-88.1)$ & $93.2(90.4-95.4)$ & $79.3(71.8-85.6)$ & $94.3(91.6-96.2)$ \\
\hline 4 & A & 91 (87.1-93.9) & $83.6(76.4-89.3)$ & $93.4(90.7-95.5)$ & $80.1(72.7-86.3)$ & $94.7(92.2-96.6)$ \\
\hline 5 & $A, V$ & $91.6(87.4-94.4)$ & $84.3(77.2-89.9)$ & 93.9 (91.2-95.9) & $81.4(74.1-87.4)$ & $94.9(92.4-96.8)$ \\
\hline
\end{tabular}

Note:- $U$ indicates unenhanced; A, arterial; $V$, venous; D, delayed.

located in the mediastinum. If a lesion was encountered in an unusual location, such as in the retropharyngeal space, the readers were asked to indicate this. Note that the quadrants were named on the basis of anatomic position on imaging and not the embryologic origin of the gland. This classification is described as "localization" below. Because there is potential for confusion due to the difference in radiographic anatomic location and embryologic anatomic location, each lesion was also classified on the basis of its general location, either left, right, or mediastinal. This classification is termed "lateralization" below.

The attenuation of each lesion was measured in Hounsfield units for each phase of contrast. For comparison, the Hounsfield unit of a representative lymph node and thyroid tissue was measured for each phase of contrast. To measure the attenuation, we coregistered the 4 phases. Then, a region of interest was drawn to incorporate as much of the suspected lesion as possible while excluding the edge of the tissue to limit extraneous pixel values due to volume averaging. The region of interest was copied to the other 3 phases, and the accuracy of the region-of-interest positioning was verified.

The surgical records were reviewed to identify the locations of the abnormal glands. Intraoperative parathyroid hormone levels and pathologic analysis were used to verify that the suspected lesion was, in fact, an abnormal gland (adenoma or hyperplasia).

\section{Statistical Analyses}

Analysis of Measurement Agreement. The surgical findings (pathologically abnormal gland, the removal of which resulted in a drop in serum parathormone levels) were considered the reference standard. Measurement agreement was evaluated for both the neuroradiologists' interpretations of the presence/absence of parathyroid lesions by using localization categorization and for neuroradiologists' interpretations of the presence/absence of parathyroid lesions by using lateralization categorization. Measurement agreement was assessed in terms of the sensitivity and the specificity of the neuroradiologically based interpretations relative to the surgical findings and in terms of the positive predictive value (PPV) and the negative predictive value (NPV) of the neuroradiologically based interpretations. Ninety-five percent confidence interval construction for each of the aforementioned summary measures was based on the exact methods of Agresti and Coull. ${ }^{11}$ Area under the receiver operating curve was estimated on the basis of the predictions of binomial generalized estimating equation regression models (On-line Tables 1 and 3).

Analysis of Neuroradiologic Interpretation Accuracy. The accuracy of the neuroradiologists' interpretations of the presence/absence of parathyroid lesions by using localization and lateraliza- tion categorization in relationship to the surgical findings was assessed by binomial generalized estimating equation regression models. ${ }^{12}$ Two regression analyses were conducted. One regression analysis evaluated the accuracy of the neuroradiologists' localization categorizations, while the other evaluated the accuracy of the neuroradiologists' lateralization categorizations. For each regression analysis, the outcome variable was a binary indicator variable that was assigned the value 1 if the neuroradiologist's parathyroid lesion interpretation (present, absent) agreed with the surgical findings, and the value zero was assigned if the 2 findings disagreed. Each regression model included a single categoric predictor variable that identified the $54 \mathrm{D}$ CT sequence subsets (Table 1). Because the 4 neuroradiologists' parathyroid lesion interpretations could not be assumed to be independent for the same patient, the variance-covariance matrices of the generalized estimating equation regression models were estimated via the Huber-White sandwich variance-covariance estimator to account for inter-reader parathyroid lesion-interpretation correlation in the hypothesis testing and the confidence interval construction processes. ${ }^{13}$ Accuracy was defined as the percentage agreement between the neuroradiologists' interpretations and the surgical findings with respect to the presence/absence of parathyroid lesions. Ninety-five percent confidence interval construction for neuroradiologic interpretation accuracy was based on the generalized estimating equation version of the Wald confidence interval construction method. The GENMOD Procedure of the statistical software package SAS, Version 9.3 (SAS Institute, Cary, North Carolina) was used to conduct the aforementioned generalized estimating equation analyses.

Interreader Agreement. Interreader agreement was assessed in 2 ways for both the localization categorizations and the lateralization categorizations. "Complete" interreader agreement required that all 4 readers have the same interpretation with respect to the presences/absence of parathyroid lesions, while "consensus" interreader agreement required that at least 3 of the 4 readers have the same interpretation with respect to the presence/absence of parathyroid lesions. For both criteria, interreader agreement was summarized as the percentage of image assessments in which the criterion was met.

See On-line Tables 1-6 for additional information.

\section{RESULTS}

\section{Patients}

Twenty-four of the 29 patients in this study were female. The mean age was $56.1 \pm 12.8$ years with a minimum of 14 years and a maximum of 74 years. The median age was 57 years. The 25 th percentile age was 50 years, and the 75 th percentile age was 67 years.

AJNR Am J Neuroradiol 35:1959-64 Oct 2014 www.ajnr.org 
Table 3: Results of lateralization analysis by sequence combination

\begin{tabular}{|c|c|c|c|c|c|c|}
\hline Week & Sequences & $\begin{array}{c}\text { Accuracy \% } \\
(95 \% \mathrm{Cl})\end{array}$ & $\begin{array}{c}\text { Sensitivity \% } \\
(95 \% \mathrm{CI})\end{array}$ & $\begin{array}{c}\text { Specificity \% } \\
\text { (95\% CI) }\end{array}$ & $\begin{array}{c}\text { PPV \% } \\
(95 \% \mathrm{CI})\end{array}$ & $\begin{array}{l}\text { NPV \% } \\
(95 \% \mathrm{CI})\end{array}$ \\
\hline 1 & $U, A$ & $91.1(85.8-94.6)$ & $89.5(82.7-94.3)$ & $92.0(87.6-95.2)$ & $86.0(78.8-91.5)$ & $94.1(90.1-96.8)$ \\
\hline 2 & $\mathrm{U}, \mathrm{A}, \mathrm{V}$ & 91.7 (87.1-94.7) & 91.9 (85.7-96.1) & $91.5(87.1-94.8)$ & 85.7 (78.6-91.2) & 95.3 (91.6-97.7) \\
\hline 3 & $U, A, V, D$ & $90.8(86.5-93.8)$ & $90.3(83.7-94.9)$ & $91.1(86.5-94.5)$ & $84.8(77.6-90.5)$ & $94.4(90.5-97.1)$ \\
\hline 4 & A & 90.5 (85.4-94) & 88.7 (81.8-93.7) & 91.5 (87.1-94.8) & $85.3(78-90.8)$ & $93.6(89.5-96.5)$ \\
\hline 5 & $A, V$ & 92. (87.5-95.3) & 92.7 (86.7-96.6) & 92 (87.6-95.2) & $86.5(79.5-91.8)$ & 95.8 (92.2-98.1) \\
\hline
\end{tabular}

Note:- $U$ indicates unenhanced; A, arterial; $V$, venous; $D$, delayed.

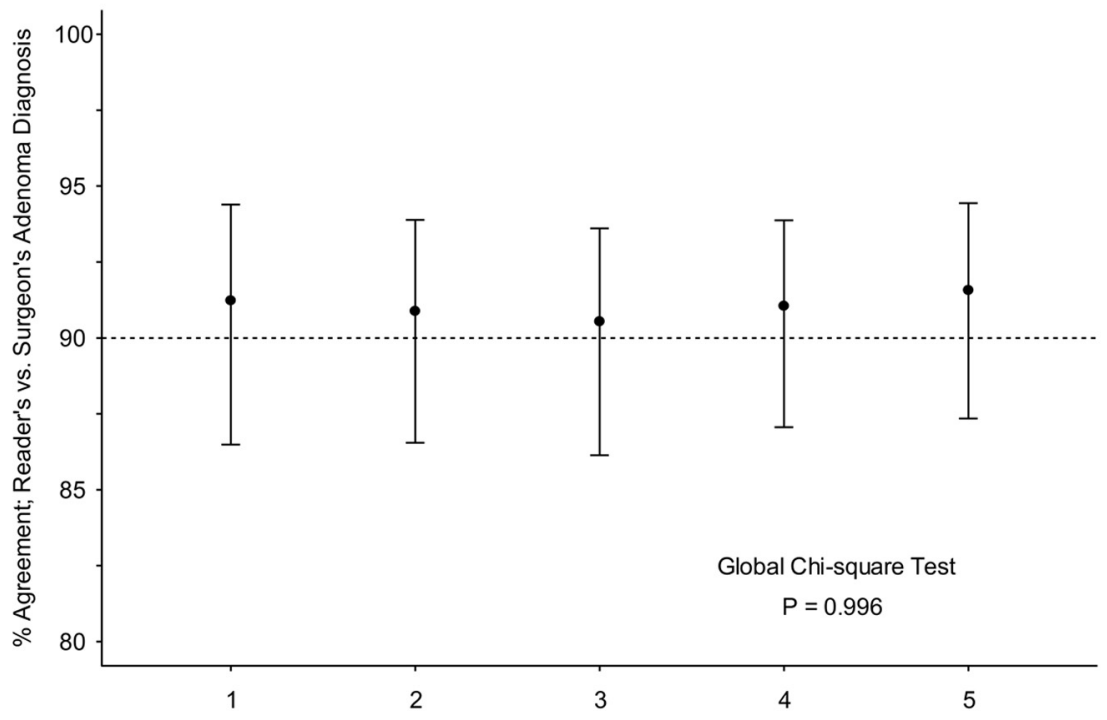

A

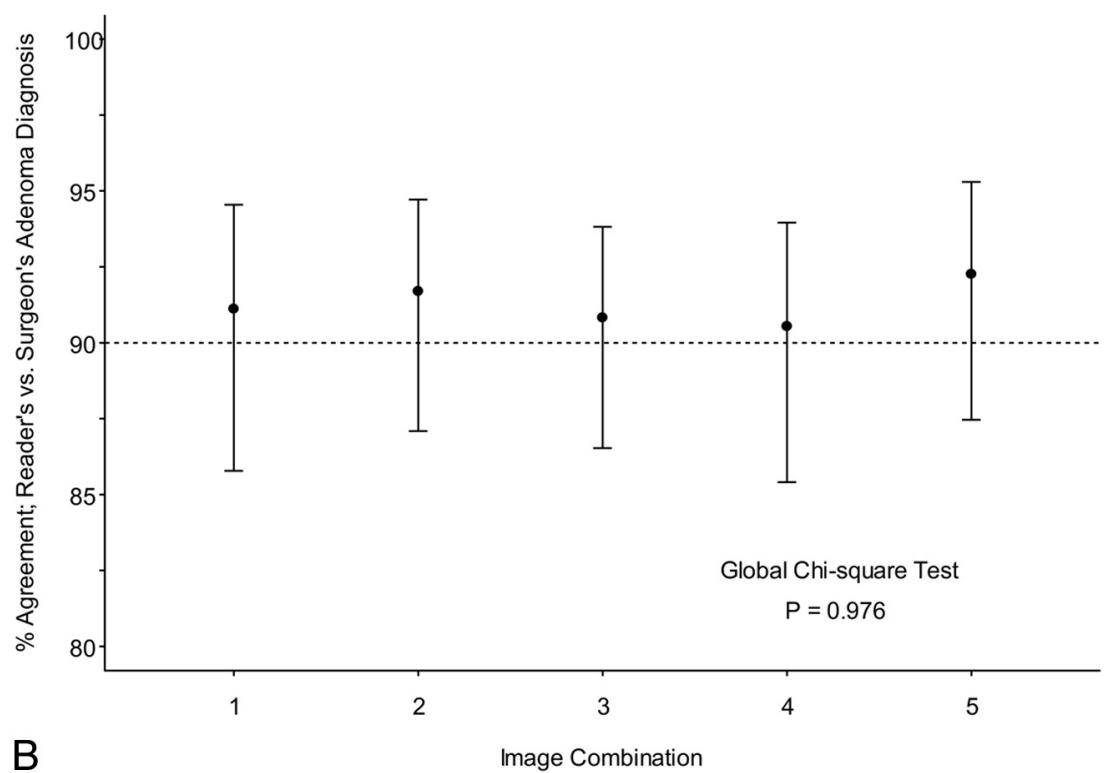

FIG 1. A, Accuracy of radiologic interpretation of parathyroid lesions by using localization categorization. $B$, Accuracy of radiographic interpretation of parathyroid lesions by using lateralization categorization. Imaging combinations are given in Table 1.

\section{Surgical/Pathologic Analysis}

Of the 29 patients, 3 had no parathyroid lesion at surgery. Five patients had multiglandular disease, 3 of which were hyperplastic and 2 of which were adenomatous. Twenty-one patients had single glandular disease: Twenty were adenomatous and 1 was hyperplastic. The mean weight of the abnormal glands was $539 \mathrm{mg}$, (median, $520 \pm 429.8 \mathrm{mg}$; range, 6-2200 mg).

\section{D CT Interpretation}

Sensitivities for identifying the specific location of a parathyroid lesion were $>80 \%$ for each of the imaging combinations, while the specificities were relatively consistent between $93 \%$ and $94 \%$ as seen in Table 2 . The lateralization analysis yielded improved sensitivities, ranging from $88.7 \%$ to $92.7 \%$ as seen in Table 3. However, the specificity for lateralization dipped to $91.1 \%-92 \%$ because of the $40 \%$ decrease in possible locations where an abnormal gland could be classified. Regardless of whether one used the localization or lateralization classification, there was no statistically significant difference between the diagnostic assessment for each combination of phases and the surgical findings with $P$ values of .996 and .976 , respectively, as seen in Fig 1 . The consistency from week to week was, in part, due to a complete interreader agreement of $78 \%$ for localization and $77 \%$ for lateralization and a consensus interreader agreement of $95 \%$ by localization and $95 \%$ for lateralization (On-line Tables 1-6, On-line Figs 1 and 2).

Reader-to-surgeon agreement by using localization categorization ranged from $85.2 \%$ to $90.2 \%$, with an average of $88.1 \%$ (Fig $1 A$ ). For lateralization, agreement ranged from $84.6 \%$ to $91.7 \%$, with an average of $87.7 \%$ (Fig $1 B$ ). This agreement is summarized in Tables 2 and 3.

As seen in Fig 2, the attenuation of parathyroid lesions was similar to that of lymph nodes on noncontrast imaging. On administration of intravenous contrast, there is a greater increase in attenuation in the parathyroid lesions compared with lymph nodes, 108 and $32 \mathrm{HU}$, respectively. During the venous phase, the contrast in the parathyroid lesions begins to wash out, while the contrast of lymph nodes continues to increase. On delayed imaging, the attenuation of parathyroid lesions approaches that of lymph nodes.

\section{DISCUSSION}

Our data indicate that the accuracy, sensitivity, and specificity for localization and lateralization of the arterial phase alone were 

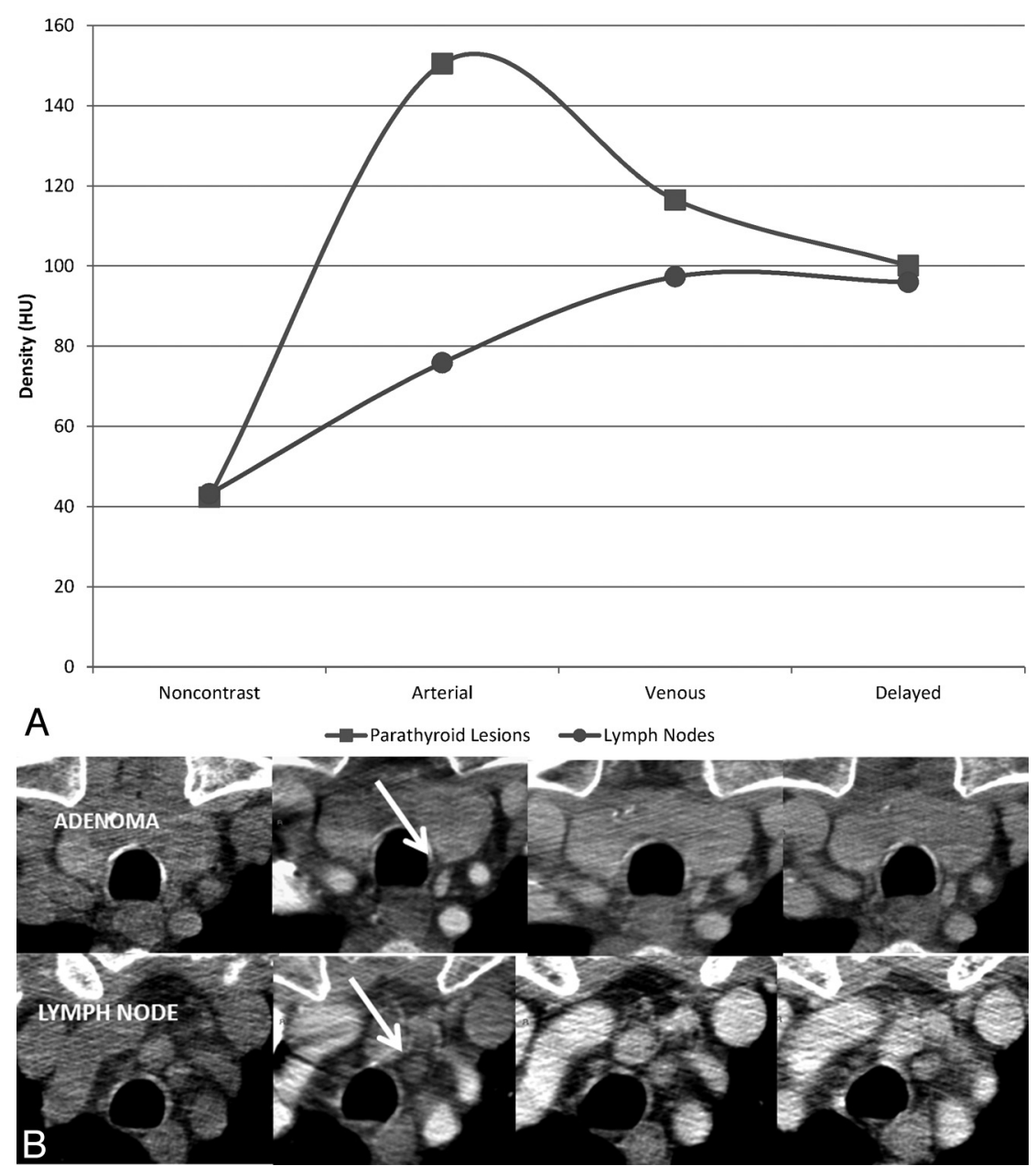

FIG 2. Comparison of the attenuation of true-positive parathyroid lesions and lymph nodes across the 4 phases of contrast. $A$, The time-attenuation curve illustrates that the greatest difference in attenuation between a parathyroid adenoma and a lymph node is in the arterial phase. In the example in $B$, showing axial unenhanced, arterial, venous, and delayed phase CT images from left to right, note that the parathyroid adenoma enhances intensely in the arterial phase, while the lymph node does not (arrow). The 2 demonstrate similar densities in the venous and delayed phases.

comparable with the full complement of 4 phases in our cohort of patients. Our sensitivities for lateralization and localization are also similar to those reported by Rodgers et $\mathrm{al}^{3}$ (sensitivity for lateralization, $88 \%$; for localization, $70 \%$ ), Chazen et $\mathrm{al}^{5}$ (93\%, $92 \%)$, Starker et $\mathrm{al}^{6}(93.9 \%, 85.7 \%)$, and Beland et $\mathrm{al}^{7}$ (localization $82 \%$ ). They are also comparable with a recent article by Hunter et al, ${ }^{14}$ who, in a study of 4D CT in 143 patients with single-gland disease, reported lateralization and localization accuracies of $93.7 \%$ and $86.6 \%$, respectively. The high accuracy of the arterial phase alone is not a surprising finding, given that the greatest difference in attenuation between an adenoma and small lymph nodes is in the arterial phase. This is illustrated in the time-attenuation curve in Fig 2.

All the parathyroid lesions in our series demonstrated arterial phase hyperenhancement. Most adenomas share 2 distinctive features, rapid arterial phase enhancement and location in typical anatomic areas, both of which can be characterized on arterial phase imaging. There may, however, be instances in which additional phases are necessary. For example, a case may be made for an unenhanced phase to enable differentiation of an intrinsically hyperattenuated exophytic thyroid nodule from an enhancing parathyroid lesion. An unenhanced phase would also be of use in patients whose adenomas are not separable by a fat plane from the thyroid. Unenhanced phases would not be expected to be helpful if the thyroid itself is diseased and is not inherently hyperattenuated, a phenomenon not infrequently encountered. In addition, in patients with a history of thyroidectomy, this phase could be omitted. Additional phases are likely of value in the detection of intrathyroidal adenomas. These, however, are uncommon and account for $<1 \%$ of all adenomas. ${ }^{15}$

Hunter et $\mathrm{al}^{16}$ proposed a logistic regression model that incorporates information from a triple-phase 4D CT scan of the neck to provide improved discriminatory ability compared with a dual-phase 4D CT scan when used to distinguish parathyroid adenomas and local mimics. Their data indicate that a model using an unenhanced, arterial phase had an accuracy of $94.7 \%$ compared with $96.9 \%$ in a model built from 3 phases. While statistically significant and interesting, the clinical utility of a $1.9 \%$ gain in accuracy would have to be evaluated in a prospective study. Such a model also has the limitation of requiring a stand-alone calculator, an endeavor that is not trivial in routine clinical practice. Furthermore, not all parathyroid adenomas enhance intensely in the arterial phase. These include lipoadenomas and cystic adenomas, both rare lesions. ${ }^{17,18}$ It may be argued that the fat content of the former and the cystic nature of the latter should be detectable in any phase and the performance of multiple phases confers no benefit.

As discussed in a recent review of the subject, concerns about the radiation exposure associated with $4 \mathrm{D}$ CT do exist. ${ }^{19}$ Mahajan et $\mathrm{al}^{20}$ calculated a $10.4-\mathrm{mSv}$ patient effective dose for $4 \mathrm{D} \mathrm{CT}$, while in a study by Madorin et al, ${ }^{21}$ it was $5.56 \mathrm{mSv}$, highlighting that the differences in radiation dosage between these studies reflect the variability in dynamic parathyroid CT protocols between institutions. However, although the effective dose to the thyroid is significantly higher with 4D CT than with scintigraphy, the lifetime attributable risk of thyroid cancer in the typical demographic that presents with hyperparathyroidism (women older than 50 years) is 3 times lower than the 2009 population incidence of thyroid cancer (14 per 100,000). ${ }^{22}$ The thyroid cancer risk does, however, exceed the current thyroid cancer incidence in younger patients (females 30 years of age or younger or males 20 years of age or younger). It is, therefore, prudent to use 4D CT judiciously, 
especially in younger patients. Substituting the 4D study with a single arterial phase in such patients can reduce radiation dose without substantially sacrificing accuracy.

Our study has some important limitations. The sample size (29 patients) is relatively small, and our readers were asked to interpret the same studies 5 times, though in varying combinations of phases. This requirement may impart an element of memory bias, which would favor interpretations performed in the later weeks of the study. We attempted to minimize this by allowing at least 1 week between each assessment and by ensuring that the patients were de-identified and the reading order was rescrambled each week. Additionally, we structured the study so that those phases that were hypothesized to yield the least confidence (single-phase interpretation, 2-phase interpretation, and so forth) were performed at the beginning of the study to ensure that memory bias would be less of an issue while interpreting these phases. Also due to the small sample size, factors such as streak artifacts from the contrast bolus, distorted anatomy due to previous surgery, and slips and remnants of thyroid tissue were not encountered to a degree significant enough to confound interpretation.

\section{CONCLUSIONS}

Our preliminary data suggest that in routine practice, multiple phases may not be necessary for the localization of parathyroid lesions. In most cases, a single arterial phase CT study may be sufficient, conveying the important advantage of reduction in radiation dose, especially in younger patients. There are instances in which additional phases may be of benefit, but their relatively low prevalence may not warrant subjecting every patient with a suspected parathyroid adenoma to the full complement of phases and the radiation dose that this entails. A full 4D CT study could be considered a problem-solving tool in those cases in which single-phase imaging is equivocal.

Disclosures: Max Wintermark-UNRELATED: Consultancy: Biogen Idec, ${ }^{*}$ St. Jude Medical,* Grants/Grants Pending: GE Healthcare, Philips Healthcare. *Money paid to the institution.

\section{REFERENCES}

1. Rodgers SE, Lew JI, Solorzano CC. Primary hyperparathyroidism. Curr Opin Oncol 2008;20:52-58

2. DeLellis RA, Mazzaglia P, Mangray S. Primary hyperparathyroidism: a current perspective. Arch Pathol Lab Med 2008;132:1251-62

3. Rodgers SE, Hunter GJ, Hamberg LM, et al. Improved preoperative planning for directed parathyroidectomy with 4-dimensional computed tomography. Surgery 2006;140:932-40, discussion 940-41

4. Gotway MB, Higgins CB. MR imaging of the thyroid and parathyroid glands. Magn Reson Imaging Clin N Am 2000;8:163-82, ix

5. Chazen JL, Gupta A, Dunning A, et al. Diagnostic accuracy of 4D-CT for parathyroid adenomas and hyperplasia. AJNR Am J Neuroradiol 2012;33:429-33

6. Starker LF, Mahajan A, Bjorklund $\mathrm{P}$, et al. 4D parathyroid CT as the initial localization study for patients with de novo primary hyperparathyroidism. Ann Surg Oncol 2011;18:1723-28

7. Beland MD, Mayo-Smith WW, Grand DJ, et al. Dynamic MDCT for localization of occult parathyroid adenomas in 26 patients with primary hyperparathyroidism. AJR Am J Roentgenol 2011;196:61-65

8. Linda DD, $\mathrm{Ng} \mathrm{B}$, Rebello R, et al. The utility of multidetector computed tomography for detection of parathyroid disease in the setting of primary hyperparathyroidism. Can Assoc Radiol J 2012;63: $100-08$

9. Kutler DI, Moquete R, Kazam E, et al. Parathyroid localization with modified 4D-computed tomography and ultrasonography for patients with primary hyperparathyroidism. Laryngoscope 2011;121: $1219-24$

10. Harari A, Zarnegar R, Lee J, et al. Computed tomography can guide focused exploration in select patients with primary hyperparathyroidism and negative sestamibi scanning. Surgery 2008;144:970-76, discussion 976-79

11. Agresti A, Coull BA. Approximate is better than "exact" for interval estimation of binomial proportions. The American Statistician 1998;52:119-26

12. Hardin JW, Hilbe JM. Generalized Estimating Equations. Boca Raton: Chapman and Hall/CRC; 2003

13. Huber PJ. The behavior of maximum likelihood estimates under nonstandard conditions. In: Le Cam LM, Neyman J, eds. Proceedings of the Fifth Berkeley Symposium on Mathematical Statistics and Probability. Vol 1. Berkeley: University of California Press; 1967

14. Hunter GJ, Schellingerhout D, Vu TH, et al. Accuracy of four-dimensional CT for the localization of abnormal parathyroid glands in patients with primary hyperparathyroidism. Radiology 2012;264: 789-95

15. Mazeh H, Kouniavsky G, Schneider DF, et al. Intrathyroidal parathyroid glands: small, but mighty (a Napoleon phenomenon). Surgery 2012;152:1193-200

16. Hunter GJ, Ginat DT, Kelly HR, et al. Discriminating parathyroid adenoma from local mimics by using inherent tissue attenuation and vascular information obtained with four-dimensional CT: formulation of a multinomial logistic regression model. Radiology 2014;270:168-75

17. Chow LS, Erickson LA, Abu-Lebdeh HS, et al. Parathyroid lipoadenomas: a rare cause of primary hyperparathyroidism. Endocr Pract 2006;12:131-36

18. Sillery JC, DeLone DR, Welker KM. Cystic parathyroid adenomas on dynamic CT. AJNR Am J Neuroradiol 2011;32:E107-09

19. Hoang JK, Sung WK, Bahl M, et al. How to perform parathyroid 4D CT: tips and traps for technique and interpretation. Radiology 2014;270:15-24

20. Mahajan A, Starker LF, Ghita M, et al. Parathyroid four-dimensional computed tomography: evaluation of radiation dose exposure during preoperative localization of parathyroid tumors in primary hyperparathyroidism. World J Surg 2012;36:1335-39

21. Madorin CA, Owen R, Coakley B, et al. Comparison of radiation exposure and cost between dynamic computed tomography and sestamibi scintigraphy for preoperative localization of parathyroid lesions. JAMA Surg 2013;148:500-03

22. Surveillance Research Program. National Cancer Institute SEER ${ }^{\star}$ Stat software. Version 1983-2009. seer.cancer.gov/seerstat. Accessed October 1,2013 